\title{
Combined Effect of Conservation of Energy Resources Strategies and Hatha Yoga on the Fatigue Severity in Patients with Rheumatoid Arthritis
}

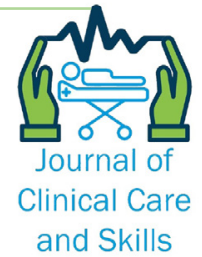

\section{ARTICLE INFO}

\section{Article Type}

Original Research

\section{Authors}

Gholami Kalus M. ${ }^{1} M S C$ Zarei Nejad M. ${ }^{2} M D$,

Malekzadeh M. ${ }^{3} \mathrm{PhD}$,

Hashemi Mohamadabad N. ${ }^{4} M D$,

Najafi Doulatabad S. ${ }^{* 5} \mathrm{PhD}$

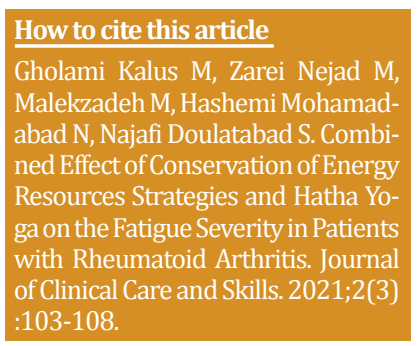

${ }^{1}$ Student Research Committee, Yasuj University of Medical Sciences, Yaרsuj, Iran ${ }^{2}$ Department of Rheumatology, Faculty of Medicine, Yasuj University of Medical Sciences, Yasuj, Iran

${ }^{3}$ Social Determinants of Health Research Center, Yasuj University of Medical Sciences, Yasuj, Iran

${ }^{4}$ Department of Psychiatry, Faculty of Medicine, Yasuj University of Medical Sciences, Yasuj, Iran

${ }^{5}$ Medical-Surgical Nursing Department, School of Nursing, Yasuj University of Medical Sciences, Yasuj, Iran

\section{*Correspondence}

Address: Medical-Surgical Nursing Department, School of Nursing, Yasuj University of Medical Sciences, Yasuj, Iran. Postal Code: 7591994799.

Phone: +98 (74) 33234115

Fax: +98 (74) 33234115

shahlaiss@yahoo.com

\section{Article History}

Received: February 28, 2021

Accepted: June 30, 2021

ePublished: September 11, 2021

\section{A B S T R A C T}

Aims Rheumatoid arthritis is the most common inflammatory arthritis in adults, and fatigue is one of its most common symptoms. This study aimed to determine the effect of combining conservation of energy resources strategies and Hatha yoga on fatigue severity in patients with rheumatoid arthritis.

Methods This clinical trial study was carried out on all eligible patients with rheumatoid arthritis referred to Shahid Beheshti Hospital and private clinics in Yasouj, Iran, in 2020-2021. Seventy-five patients were selected by convenience sampling method and then randomly assigned to three intervention groups (energy saving strategies, Hatha yoga, combined energy-saving strategy, and Hatha yoga). Data were collected through the Multidimensional Fatigue Inventory questionnaire in three intervention groups in the first session before and after the intervention. Data were analyzed using SPSS 25 software through one-way analysis of variance.

Findings There was no significant difference in the mean score of fatigue severity between study groups before the intervention $(\mathrm{p}<0.05)$. A significant decrease was observed in the mean score of fatigue ( $p>0.001)$ in the combined methods of Hatha yoga and conservation of energy resources strategies $(32.3 \pm 78.76)$ than Hatha Yoga $(38.28 \pm 2.66)$ and conservation strategies of energy resources groups (42.52 \pm 3.83$)$.

Conclusion Conservation of energy resources, Hatha yoga and a combination of conservation of energy resources, and Hatha yoga, improved fatigue severity in patients with rheumatoid arthritis, and the combined intervention was more effective than the other two methods.

Keywords Conservation of Energy Resources; Yoga; Fatigue; Rheumatoid Arthritis

\section{I T A T I O N L I N K S}

[1] Brunner \& Suddarth's textbook of medical-surgical ... [2] 2015 American college of rheumatology guideline for the treatment ... [3] The environment, geo-epidemiology, and autoimmune disease ... [4] Role of sleep disturbance, depression, obesity, and physical ... [5] Fatigue as experienced by patients with rheumatoid arthritis (RA) ... [6] Biologic interventions for fatigue in rheumatoid ... [7] APLAR rheumatoid arthritis treatment ... [8] Complementary and alternative medicine (CAM): A ... [9] Managing fatigue with technology for individuals with ... [10] Fatigue self-management behaviors in patients with advanced... [11] Efficacy of an energy conservation course for ... [12] Effectiveness of energy conservation management on fatigue and participation ... [13] The effects of yoga compared to active and inactive ... [14] The effects of yoga on patients with mild cognitive impairment ... [15] Effects of yoga on mental and physical ... [16] Yoga for the management of pain and sleep ... [17] The effect of yoga on stress, anxiety, and ... [18] Theimpact of modified Hatha yoga on chroniclow ... [19] The Multidimensional Fatigue Inventory (MFI) ... [20] Different scales of fatigue in traffic ... [21] Effectiveness of energy conservation treatment ... [22] Effect of energy conservation strategies ... [23] Energy conservation for fatigue management in ... [24] The effects of an 8-week Hatha yoga intervention on executive ... [25] A Study to assess the effectiveness of Hatha yoga ... [26] Influence of Hatha yoga on physical activity ... [27] Age related differences of selected Hatha yoga ... [28] Effects of a 12-week hatha yoga intervention ... [29] Effect of Hatha yoga on anxiety: A meta ... [30] A clientcentred programme focusing energy ... 


\section{Introduction}

Rheumatoid arthritis is an autoimmune disease of unknown origin. The autoimmune reaction occurs mainly in the synovial tissue and produces inflammatory and destructive synovial fluid [1]. Rheumatoid arthritis is the most common inflammatory arthritis in adults [2]. The prevalence of this disease is $1 \%$ in the world, 1 to $2 \%$ in the western world, $0.35 \%$ in developing countries, and about $0.4 \%$ in Iran. Although the cause of this disease is still unknown, seven risk factors have been considered for it, including genetic predisposition, gender, age, race, socioeconomic characteristics, smoking, diet, infectious and hormonal factors [3]. It usually occurs in the age groups of 30-55, and the probability of infection in women is 3 to 1 than in men. Fatigue is one of the most common symptoms in this type of patient [4], and its study is a challenging topic worldwide. Repping et al. investigated rheumatoid arthritis in 29 patients, and their results showed that patients described the sudden and frustrating nature of rheumatoid arthritis as a frustrating and upsetting case. These patients also consider the fatigue caused by the disease to be very excruciating, so that it has negative effects on daily work, attitude, and leisure [5]. Evidence showed that fatigue results from the complex interaction of clinical factors (such as inflammation, pain, and disability), psychosocial problems (e.g., mood, beliefs, and behaviors), and personal factors (e.g., work, care responsibilities, and comorbidities) that may vary between individuals over time. These results are reflected in the evidence obtained from the systematic review of nonpharmacological and pharmacological treatments for fatigue [6]. There is no specific treatment for this disease, and disease management is one of the main aims [7].

Today, the demand for complementary medicine for the process of recovery and achieving health and well-being has increased, following the increase of chronic diseases [8]. Various strategies have been proposed to reduce patients' fatigue. One of these strategies is to use energy-saving strategies. The purpose of conservation of energy resources is to balancing rest time and activity during illness-related fatigue, realizing the value of rest, the purpose of conservation of energy resources, planning a rest period in a daytime self-care program [9]. These techniques help the individual to perform their functional and daily tasks effectively [10]. In this regard, the results of Mathiowetz et al. showed that conservation strategies of energy resources increase self-efficacy, improve quality of life, and reduce fatigue in patients with multiple sclerosis [11]. However, Blikman et al. revealed no effect on fatigue and limitations associated with multiple sclerosis ${ }^{[12] .}$ Yoga is a mental activity and psychophysical exercise with slow movements and strengthening the muscles to improve physical and mental conditions [13]. It is also recommended to strengthen muscles and balance activities in the national and global physical activity guidelines [14]. Hatha yoga exercises provide an alternative form of physical activity that may reduce stress, fatigue and facilitate recovery. Yoga can reduce mental fluctuations, stress, and fatigue, increase self-esteem and immune function, and improve heartbeat [15]. The results of a study by Ward et al. showed that a relaxation-based yoga program is effective for patients with pain and functional disability associated with rheumatoid arthritis [16]. Shohani et al., in a study, resulted that yoga has an effective role in reducing stress, anxiety, and depression [17].

Since chronic diseases such as rheumatoid arthritis affect all aspects of patients' economic, social, and emotional life of patients (depression, anxiety, and fatigue), they should be able to manage this fatigue and be ready for any change to improve their lives. These patients need to reconsider their plans and have enough rest to overcome fatigue, but it does not mean inactivity because inactivity causes fatigue and osteoarthritis. Considering that Hatha yoga is a simple form of yoga practice and there is no limit to doing it, it can be used as a complementary method to improve fatigue severity. On the other hand, there is some research on the effects of conservation techniques of energy resources on patients with chronic disorders. However, there are a few types of research in this field, and the results were sometimes contradictory, as shown in the above findings. In addition, despite the research background, no study was found on the combined effect of Hata yoga and conservation strategies of energy resources to assess fatigue and psychological well-being in these patients. So, this study aimed to determine the combined effect of energy conservation strategies and Hatha yoga on the severity of fatigue in patients with rheumatoid arthritis.

\section{Instrument and Methods}

This clinical trial study was carried out on all eligible patients with rheumatoid arthritis referred to Shahid Beheshti Hospital and private clinics in Yasouj, Iran, 2020-2021. The subjects were selected by convenience sampling method and then randomly assigned to three intervention groups: energy-saving strategies, Hatha yoga, combined energy-saving strategies, and Hatha yoga intervention). For this purpose, numbered coupons were placed in one container according to the number of people in each group. Each patient has randomly selected a coupon and participated in one of the three intervention groups. The sample size was considered to be 22 for each intervention group based on the parameters of $\alpha=0.05, \alpha=0.95, z_{1-\frac{\alpha}{2}}=1.96, \beta=0.2,1-\beta=0.8$ and $\mathrm{z}_{1}-$ $\beta=0.85$ and a similar study ${ }^{[18]}$. Also, the sample size was considered to be 25 for each group considering 
the statistical drop of $10 \%(n=75)$. The inclusion criteria were conscious desire to participate in the study, diagnosis of rheumatoid arthritis by a rheumatologist, literacy, age range of 30-55 years, having the physical and cognitive ability to participate in Hatha yoga exercises. Exclusion criteria included no restriction of motion, no active phase of the disease, and other chronic diseases. Data were collected using a demographic questionnaire including age, gender, occupation status, marital status, and the Multidimensional Fatigue Inventory questionnaire [19] to assess fatigue. The questionnaire consists of 20 items and five subscales of general fatigue ( 4 items), physical fatigue (4 items), decreased activity (4 items), decreased motivation (4 items), and mental fatigue (4 items), which the minimum and the maximum scores is 20 and 100, respectively. The items of the questionnaires were scored using a 5-points Likert scale $(1=y e s$, completely true to $5=$ no, completely false). Items 19 and 18, 17, 16, 14, 13, 10, 9, 5, 2 were scored in reverse method. To obtain the overall score of the questionnaire, the scores of all the items are added together. The total score of each domain is 420 , and the total score of fatigue, which is determined by the sum of the scores of the domains, can be 20100. A higher score indicates more fatigue in the subjects. The validity and reliability of this questionnaire were confirmed by Khani et al. [20]. In this study, the reliability of this questionnaire was 0.752 by Cronbach's alpha test.

The study was approved by the Ethics Committee of Yasouj University of Medical Sciences.

Informed consent forms were obtained from participants, emphasizing the confidentiality of participants' information, non-imposition of fees on patients, and education by the opposite gender. The subjects filled the demographic characteristics and the Multidimensional Fatigue Inventory questionnaires in three intervention groups. Then, the Multidimensional Fatigue Inventory was filled out by the subjects in the three groups immediately after the conservation of energy resources, Hatha yoga, and the combination of conservation strategies of energy resources and Hatha Yoga. The intervention was performed in a sports club. The necessary training was performed for eight weeks and two sessions per week for 45 minutes individually and according to each patient's condition in the two groups of energy resources strategies and Hatha yoga intervention groups. The intervention was provided for the subjects of energy conservation and Hatayoga groups for 2-45 minutes per week for eight weeks. The intervention was performed for the combined group for 2-90 minutes sessions per week for eight weeks, individually and according to each patient's condition. In the Hatayoga group, the intervention method was such that in the initial sessions, people were first placed in a state of Shavasana or a dead
Gholami Kalus M. et al.

body for 10 minutes, then mind control techniques were taught, and quadruple sports movements exercises were trained in a lying, sitting and standing position during 45 minutes. Mental attention was paid to the organs and respiration after a workout. In all three situations, the workout was performed along with breathing exercises and relaxation. The exercises progressed and developed from beginner to intermediate during the following sessions. The exercise program was modified and performed at least one or two hours after breakfast or a light lunch on a yoga mat (Matt) or at least a quiet place with a soft carpet and in balanced air (neither cold nor hot) [12]. The following items, known as the four P's, were mentioned in the group of energy conservation strategies: 1- Prioritize: (1- Identify the important activities: 2- Are your friends or family able to support you?). 2- Plan implementation: (1Awareness of when you have less energy to perform tasks and keep it in mind; 2- Balance exercise with rest, schedule your plan according to your physical activity and time required for sports movements; 3 Organizing the home and workplace so that the required items are available; 4- Having a balanced diet and avoid heavy meals. Being overweight, alcoholic, and smoking can make fatigue worse). 3Peace: (1- Try to balance rest and activity and try to rest after eating; 2- Try to rest regularly during your daily activities; 3 - Try to use a notebook to do your activities daily or weekly; 4- Try to have a regular schedule and a good night's sleep). 4- Posture-: (Position: 1- Maintaining a smooth and symmetrical position; 2- If possible, work should be done sitting instead of standing; 3 - Sit in a chair or high place such as a platform. 4: Avoid excessive rotation and bending). Energy-saving strategies and Hatha yoga exercises were taught to the patients in the combined group for 90 minutes. Data were analyzed using SPSS 25 software through descriptive statistics and inferential tests with a $95 \%$ confidence level. The natural distribution of variables was investigated using the Kolmogorov-Smirnov test. One-way analysis of variance was used to compare the means of the variables. Two by two comparison of mean differences was performed using the Tukey test. Paired t-test was used for intragroup comparison.

\section{Finding}

There was no significant difference in the demographic variables of the subjects $(p>0.05)$. The average age of the subjects was $41.66 \pm 6.55$, and $82 \%$ of the subjects were female (Table 1 ).

The variables had a normal distribution according to the Kolmogorov-Smirnov test $(\mathrm{p}<0.05)$. There was no significant difference between the groups in terms of fatigue variable before intervention $(\mathrm{p}=0.82)$, but there was a significant difference after intervention ( $<<0.05$ : Table 2). 
Table 1) Demographic characteristics of the subjects in different study groups ( $\mathrm{n}=75$ )

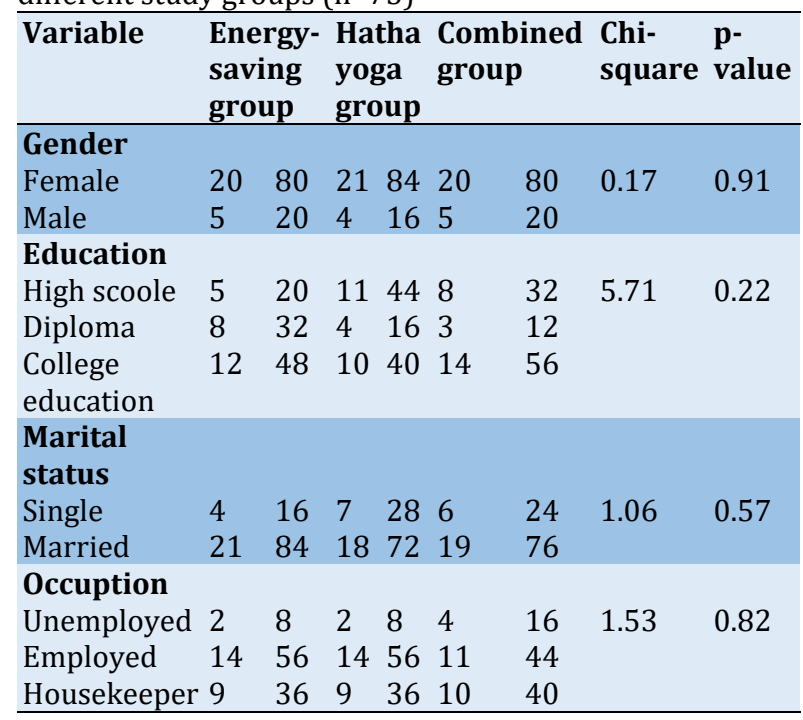

Table 2) Comparison of statistical mean of the fatigue variable between the three groups before and after intervention

\begin{tabular}{llll}
\hline Group & Pre-test & Post-test & p-value \\
\hline Energy-saving & $75.96 \pm 7.51$ & $42.52 \pm 3.83$ & 0.001 \\
Hatha yoga & $75.36 \pm 4.89$ & $38.28 \pm 2.66$ & 0.001 \\
Combined & $74.92 \pm 5.15$ & $32.48 \pm 3.76$ & 0.001 \\
p-value & 0.82 & 0.001 & - \\
\hline
\end{tabular}

The Tukey post hoc test was used to determine the differences between the groups, and it was found that there was a significant difference between the energy-saving and combined groups and between the combined and Hatha yoga groups $(\mathrm{p}<0.001)$. Also, there was a significant difference between the Hatha yoga and energy-saving groups $(\mathrm{p}<0.001)$.

\section{Discussion}

This study aimed to determine the effect of combining energy conservation strategies and Hatha yoga on fatigue severity in patients with rheumatoid arthritis. The results showed that the intervention of energy-saving, Hatha yoga exercises, and the group of combined energy-saving strategies and Hatha yoga significantly reduced the patient's fatigue with rheumatoid arthritis. The results showed a reduction in fatigue due to energy-saving strategies in patients with rheumatoid arthritis; however, this reduction was lower than the two groups of Hatha yoga and combined Hatha yoga and energy-saving groups. Blickman et al. found that treatment with conservation of energy resources could reduce the effects of fatigue and improve the three subscales of quality of life, social functioning, and mental health in patients with multiple sclerosis in the short term. The results of the Blickman et al. study are in accordance with the present study's findings [21].

As the results of this study, El Fadeel \& El-Deen, in a study, showed that energy conservation strategies could reduce fatigue and improve the level of independence of patients with systemic lupus erythematosus during daily activities [22]. In the present study, similar results have been obtained due to the similarity of rheumatism and systemic lupus erythematosus and complication of fatigue in both diseases. García Jalón et al., in a random controlled trial study, showed that an energy conservation program is effective in multiple sclerosis control [23]. The similarity of the results of these studies with the present study indicates the effect of energy conservation strategies on fatigue of chronic patients. Hatha yoga intervention has also improved fatigue in patients with rheumatoid arthritis; although this reduction was more than the effect of energy conservation intervention, it was less than the effect of the combined intervention. As the present study results, Gothe et al. aimed to determine the effect of 8 weeks of Hatha yoga intervention on organizational performance in the elderly, showed considerable improvement in the elderly performance after the training [24]. In both studies, the intervention variable, the duration of the exercises, and the type of intervention were the same and had similar results. Similar to the results of this study, Ramya, in a study, found that Hatha yoga is an effective intervention in reducing fatigue and insomnia in patients on hemodialysis [25]. Although the intervention in the above study was performed in a shorter time and hemodialysis patients have different conditions from patients with rheumatism, in both studies, Hatayoga reduced fatigue following energy recovery in each disease. Van Puymbroeck et al., in a study aimed at determining the effect of Hatha yoga on the limitation of physical activity, fitness, and body image of breast cancer survivors, showed positive changes in body image, physical activity limitations, and physical fitness for participants.

Hatha yoga may reduce physical activity limitations and improve fitness in breast cancer survivors [26]. The results of this study are by the research of Van Puymbroeck et al. Hatha yoga, which is a combination of static and dynamic stretching exercises, is a safe and appropriate way to improve the overall flexibility of individuals, which improves the flexibility of the neck, back, and hamstring muscle [27]. Therefore, Hatha yoga increased the performance of the elderly in the mentioned study. Hatha yoga has been considered by mental health professionals in improving mood outcomes, and studies show that Hatayoga is an adjunctive therapy for various mood disorders [28]. In this regard, Andreoli et al. showed that Hatha yoga is a promising way to treat anxiety [29].

In the present study, the combined intervention of Hatha yoga and energy conservation strategies reduced fatigue in patients with rheumatoid arthritis more than the two interventions of energy conservation strategies and Hatha yoga, separately. Interventions of energy conservation strategies lead to changes in people's daily activities, including 
increased participation, independence, and conscious choice to prioritize or refrain from certain activities, and all of these are natural effects of using energy conservation strategies [30]. Therefore, it can affect patients' fatigue. Hatha yoga also shrinks flexibly with age due to the increasing stiffness of the soft tissue structures of the joint capsule, muscles and facies, tendons, ligaments, and skin of various joints, leading to physical problems such as pain syndrome or balance disorders. Hatha yoga with flexibility also decreases with aging due to the increase in stiffness and soft tissue structures of the joint capsule, facial muscle, and area, tendons, ligaments, and skin of various joints. Some benefits related to patient functions such as range of motion and fatigue can be found after yoga intervention, which partly explains its effect on reducing fatigue. Due to the positive effects of Hatha yoga and energy conservation strategies, the combination of the two methods has further improved fatigue in patients with rheumatoid arthritis.

Although the findings of this study indicate a reduction in fatigue in patients with rheumatoid arthritis, there were some limitations. The limitations of this study were the non-acceptance of cooperation by patients due to the Covoid-19 pandemic and patients' stress which was beyond the researcher's control. Also, the large number of questionnaire items led to the prolongation of its implementation, which may have affected the accuracy of the participants' answers. According to the findings of this study, it is suggested to study other patients with fatigue problems (patients with multiple sclerosis, etc.).

\section{Conclusion}

The combined method of energy conservation strategies and Hatha yoga has improved the severity of fatigue in patients with rheumatoid arthritis. Therefore, it can be concluded that the combined implementation of energy conservation strategies and Hatha yoga on the severity of fatigue improves the severity of fatigue in these patients and will lead to a better quality of life and physical and mental health. Also, due to the importance of training and the type of training for these people, it is recommended that health team members consider these training methods for patients with rheumatoid arthritis. In addition, these exercises can be done individually at home.

Acknowledgments: We thank the Vice-Chancellor for Research of Yasouj University of Medical Sciences for this study's financial and spiritual support and the patients who participated in this study.

Ethical Permissions: This study has been approved by the Ethics Committee of the Vice Chancellor for Research and Technology of Yasouj University of Medical Sciences with the ethics code of 133.1398.IR.YUMS.REC.
Conflicts of Interests: This research is retrieved from the master's thesis of the first writer.

Authors' Contribution: Gholami M. (First Author), Introduction Writer/Original Researcher (40\%); Zarei Nejad M. (Second Author), Introduction Writer/Discussion Writer (10\%); Malekzadeh M. (Third Author), Introduction Writer/Methodologist (15\%); Hashemi Mohamadabad N. (Forth Author), Introduction Writer/Discussion Writer (10\%); Najafi Doulatabad S. (Fifth Author), Introduction Writer/Statistical Analyst/Discussion Writer (25\%)

Funding/Support: This study is supported by Yasuj University of Medical Sciences

\section{References}

1- Hinkle JL, Cheever KH. Brunner \& Suddarth's textbook of medical-surgical nursing. Philadelphia: Wolters Kluwer Health; 2013.

2- Singh JA, Saag KG, Bridges Jr SL, Akl EA, Bannuru RR, Sullivan MC, et al. 2015 American college of rheumatology guideline for the treatment of rheumatoid arthritis. Arthritis Rheumatol. 2016;68(1):1-26.

3- Tobon GJ, Youinou P, Saraux A. The environment, geoepidemiology, and autoimmune disease: Rheumatoid arthritis. J Autoimmun. 2010;35(1):10-4.

4- Katz P, Margaretten M, Trupin L, Schmajuk G, Yazdany J, Yelin E. Role of sleep disturbance, depression, obesity, and physical inactivity in fatigue in rheumatoid arthritis. Arthritis Care Res. 2016;68(1):81-90.

5- Repping-Wuts H, Uitterhoeve R, Van Riel P, van Achterberg T. Fatigue as experienced by patients with rheumatoid arthritis (RA): A qualitative study. Int J Nurs Stud. 2008;45(7):995-1002.

6- Almeida C, Choy EH, Hewlett S, Kirwan JR, Cramp F, Chalder $\mathrm{T}$, et al. Biologic interventions for fatigue in rheumatoid arthritis. Cochrane Database of Syst Rev. 2016;2016(6):008334.

7- Lau CS, Chia F, Harrison A, Hsieh TY, Jain R, Jung SM, et al. APLAR rheumatoid arthritis treatment recommendations. Int J Rheum Dis. 2015;18(7):685-713.

8- Idiana I, Hadi AHA, Keng SL. Complementary and alternative medicine (CAM): A comparative study between nursing and medical students. Educ Med J. 2012;4(2):94-8. 9- Grant J, Gamueda MA, Ortega A, Song J. Managing fatigue with technology for individuals with multiple sclerosis [dissertation]. Ottawa: Dominican University College; 2018.

10- Chan R, Yates P, McCarthy AL. Fatigue self-management behaviors in patients with advanced cancer: A prospective longitudinal survey. Oncol Nurs Forum. 2016;43(6):76271.

11- Mathiowetz V, Matuska KM, Murphy ME. Efficacy of an energy conservation course for persons with multiple sclerosis. Arch Phys Med Rehabil. 2001;82(4):449-56.

12- Blikman LJ, Meeteren JV, Twisk JW, De Laat FA, De Groot $\mathrm{V}$, Beckerman $\mathrm{H}$, et al. Effectiveness of energy conservation management on fatigue and participation in multiple sclerosis: A randomized controlled trial. Mult Scler. 2017;23(11):1527-41.

13- Sivaramakrishnan D, Fitzsimons C, Kelly P, Ludwig K, Mutrie N, Saunders DH, et al. The effects of yoga compared to active and inactive controls on physical function and health related quality of life in older adults-systematic review and meta-analysis of randomised controlled trials. Int J Behav Nutr Phys Act. 2019;16(1):33. 
14- Brenes GA, Sohl S, Wells RE, Befus D, Campos CL, Danhauer SC. The effects of yoga on patients with mild cognitive impairment and dementia: A scoping review. Am J Geriatr Psychiatry. 2019;27(2):188-97.

15- Nayak AK. Effects of yoga on mental and physical health. Int J Phys Educ Sports Health. 2016;3(5):268-70.

16- Ward L, Stebbings S, Athens J, Cherkin D, Baxter GD. Yoga for the management of pain and sleep in rheumatoid arthritis: A pilot randomized controlled trial. Musculoskeletal Care. 2018;16(1):39-47.

17- Shohani M, Badfar G, Nasirkandy MP, Kaikhavani S, Rahmati S, Modmeli Y, et al. The effect of yoga on stress, anxiety, and depression in women. Int J Prev Med. 2018;9:21.

18- Gatantino ML, Bzdewka TM, Eissler-Rnsso JL, Holbrook ML, Mogck EP, Geigle P, et al. The impact of modified Hatha yoga on chronic low back pain: A pilot study. Altern Ther Health Med. 2004;10(2):56-9.

19- Smets EMA, Bert Garssen B, de Bonke, de Haes JCJM. The Multidimensional Fatigue Inventory (MFI) psychometric qualities of an instrument to assess fatigue. J Psychosom Res. 1995;39(3):315-25.

20- Khani JR, Saremi M, Kavousi A, Shirzad H, Rezapour T. Different scales of fatigue in traffic policemen. Police Med. 2012;1(1):2-10. [Persian]

21- Blikman LJ, Huisstede BM, Kooijmans H, Stam HJ, Bussmann JB, Van Meeteren J. Effectiveness of energy conservation treatment in reducing fatigue in multiple sclerosis: A systematic review and meta-analysis. Arch Phys Med Rehabil. 2013;94(7):1360-76.

22- El Fadeel ES, El-Deen DS. Effect of energy conservation strategies on fatigue and daily living activities among patients with systemic lupus erythematosus. Egypt Nurs J.
2020;17(1):36-46.

23- Garcia Jalon EG, Lennon S, Peoples L, Murphy S, LoweStrong A. Energy conservation for fatigue management in multiple sclerosis: A pilot randomized controlled trial. Clin Rehabil. 2013;27(1):63-74

24- Gothe NP, Kramer AF, McAuley E. The effects of an 8week Hatha yoga intervention on executive function in older adults. J Gerontol A Biol Sci Med Sci. 2014;69(9):1109-16.

25- Ramya S. A Study to assess the effectiveness of Hatha yoga on fatigue and insomnia among patients with hemodialysis in selected hospital at Erode. Bishop's College of Nursing, Dharapuram; 2015.

26- van Puymbroeck M, Schmid A, Shinew K, Hsieh PC. Influence of Hatha yoga on physical activity constraints, physical fitness, and body image of breast cancer survivors: A pilot study. Int J Yoga Ther. 2011;21(1):49-60.

27- Halder K, Chatterjee A, Pal R, Tomer OS, Saha M. Age related differences of selected Hatha yoga practices on anthropometric characteristics, muscular strength and flexibility of healthy individuals. Int J Yoga. 2015;8(1):3746.

28- Lau C, Yu R, Woo J. Effects of a 12-week hatha yoga intervention on metabolic risk and quality of life in Hong Kong Chinese adults with and without metabolic syndrome. PLos One. 2015;10(6):0130731.

29- Andreoli G, Curtiss J, Hofmann SG, Carpenter JK. Effect of Hatha yoga on anxiety: A meta-analysis. J Evid Based Med. 2016;9(3):116-24.

30- Norberg EB, Lofgren B, Boman K, Wennberg P, Brannstrom M. A client-centred programme focusing energy conservation for people with heart failure. Scand J Occup Ther. 2017;24(6):455-67. 